\title{
In situ mechanical observations during nanoindentation inside a high-resolution scanning electron microscope
}

\author{
K.A. Rzepiejewska-Malyska, ${ }^{\text {a) }}$ G. Buerki, and J. Michler \\ Swiss Federal Laboratories for Material Testing and Research-EMPA, CH-3602 Thun, Switzerland \\ R.C. Major, E. Cyrankowski, S.A.S. Asif, and O.L. Warren \\ Hysitron, Inc., Minneapolis, Minnesota 55344
}

(Received 18 December 2007; accepted 7 April 2008)

\begin{abstract}
In nanoindentation, the occurrence of cracks, pileup, sink-in, or film delamination adds additional complexity to the analysis of the load-displacement curves. Many techniques and analysis methods have been used to extract both qualitative and quantitative information from the indentation test both during and after the test. Much of this information is obtained indirectly or may even be overlooked by current testing methods (e.g., cracks that open only during the loading cycle of the test may go unnoticed from a typical residual indentation analysis). Here we report on the development of a miniature depth-sensing nanoindentation instrument and its integration into a high-resolution scanning electron microscope. Real-time observation of the nanoindentation test via scanning electron microscopy allows for visualization and detection of certain events such as crack initiation, pileup, or sink-in, and other material deformation phenomena. Initial results from aluminum $\langle 100\rangle$ and a thin gold film $(\sim 225 \mathrm{~nm})$ are presented.
\end{abstract}

\section{INTRODUCTION}

Nanoindentation is one of the most widely used techniques for mechanical characterization of thin-film coatings and nanostructured materials. ${ }^{1}$ The standard nanoindentation test yields a force-displacement curve from which the mechanical properties of the tested material can be extracted. The onset of plastic deformation in addition to hardness and elastic modulus of the material are examples of information obtained in a standard nanoindentation experiment. Fracture behavior may be assessed by analyzing the crack length and the residual imprint left in the sample. This can give insight into the fracture toughness of the sample. Other material deformation behavior such as pileup or sink-in and thin-film delamination may be observed as well. Traditionally, these phenomena have been characterized only upon analysis performed after the nanoindentation test has been completed. Until recently, correlation of events that accompany material deformation (such as cracking, delamination, and microstructural changes) with the corresponding features in the force-displacement curve has been largely based on educated speculation. The development of quantitative, in situ, nanoindentation tools are

\footnotetext{
a) Address all correspondence to this author.

e-mail: karolina rzepiejewska@empa.ch

DOI: $10.1557 / J M R .2008 .0240$
}

a necessity for providing conclusive support for many of the proposed theories and models used to explain many of the material deformation processes that occur during the nanoindentation test.

Recent work by Minor et al. involving quantitative nanoindentation experiments inside a transmission electron microscope (TEM) illustrates the power of coupling real-time electron imaging with nanoindentation. ${ }^{2}$ This work led to new insights into the onset of plastic deformation in materials. In 1968, Gane and Bowden reported on one of the first integrations of an indentation-type device into a scanning electron microscope (SEM). ${ }^{3}$ Other groups followed along in later years with SEM integrations as well. ${ }^{4,5}$ More recently, Rabe et al. integrated a microindentation device inside a SEM. ${ }^{6}$ The in situ SEM imaging during the microindentation test provided a real-time monitor of the true contact area, pileup, and shear band formation ${ }^{7}$ throughout the indentation cycle. Another advantage of in situ SEM indentation is the capability to observe crack initiation and timecorrelate this event to the force-displacement curve. Other experiments that would be ideally suited for the SEM nanoindentation technique include bending/ deformation of nanowires as well as nanopillar and nanosphere compression among many others.

The development of a quantitative nanoindentation system integrated into a high-resolution SEM is discussed here. The design principles are somewhat similar 
to the instrument introduced elsewhere; however, there are some significant differences. ${ }^{6}$ The main differences are the electrostatic force actuation, capacitance displacement sensing, load resolution, the imaging resolution, and two possible indentation control modes (open-loop load ramp or closed-loop displacement ramp) available as well as the size of the current design. A Hysitron PicoIndenter transducer ${ }^{8}$ (Minneapolis, MN) provides high-sensitivity force-displacement data and a Hitachi S-4800 (Japan) SEM provides high-resolution imaging. This novel instrument for in situ observation has a large number of applications due to its design flexibility, which allows for sample/indenter rotation and tilt with respect to the imaging axis.

To show the potential of this technique, we performed in situ nanoindentation tests on two materials that have been widely investigated in the literature. Initial results obtained from an aluminum $\langle 100\rangle$ single crystal and a thin gold film $(\sim 225 \mathrm{~nm})$ deposited on a fused quartz substrate showed interesting material deformation and delamination behavior.

\section{EXPERIMENTAL}

\section{A. Integration of the nanoindenter into the SEM}

There are several requirements that have to be fulfilled by the instrument to be SEM compatible. Material selection is crucial for successful integration into the SEM operating environment. The used materials must be nonferromagnetic or rather cause low electromagnetic disturbance. Otherwise, they may influence the electron beam and cause distortions in the image. The operating pressure in the chamber is in the high-vacuum (HV) range. All materials used for construction were at a minimum HV if not ultrahigh-vacuum (UHV) compatible materials. The materials chosen for the support structure and main design elements were titanium, copper, and aluminum. Machinable ceramic (Macor) and vacuumcompatible epoxy were used for electrical isolation.

Modern high-resolution SEMs often have small vacuum chambers to minimize vibrations and limit the vacuum pump-down time. This puts a severe size constraint on the indenter hardware. The entire nanoindenter including motion stages, support structure, and sample stage were miniaturized so they could be inserted through the load-lock and fit inside the chamber. Due to the size restrictions of operating inside the SEM, a quantitative miniaturized force-displacement transducer was used. This transducer was originally developed for in situ TEM applications where space is even more constricted. ${ }^{2}$ The transducer is capable of independent electrostatic force actuation and capacitance-based displacement sensing. The miniature transducer comprises two sensor cores, one configured as a force actuator (Fig. 1, IIb) and the other as a displacement sensor (Fig. 1, IIa). Both

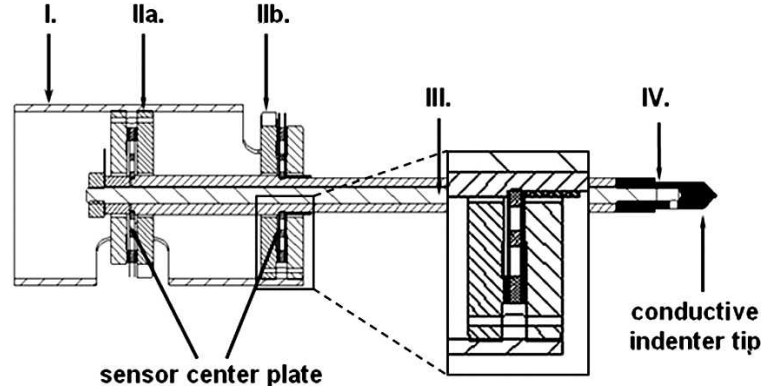

FIG. 1. Cross-sectional view of the actuatable capacitive transducer: I, transducer body; II, multiplate capacitor (inset: magnified view) (a) for displacement measurement and (b) for load actuation; III, conductive threaded rod; and IV, conductive probe.

cores are of the three-plate capacitor design, i.e., a spring suspended center plate (center electrode) occupies the space between two fixed outer plates (outer electrodes). A dielectric is used to space the plates such that they are nominally parallel. The two sensor cores are attached together by a central rod (Fig. 1, III) connecting the center plates such that they always move together. The rod (Fig. 1, III) extends from the opposite face of the center plate of one core. An indentation tip holder (Fig. 1, IV) screws onto a threaded rod that extends from the end of the central connecting rod. The connecting rod (Fig. 1, III) and the tip holder (Fig. 1, IV) are parts of a conduction path for dissipating charge from the tip when the tip is impinged on by the electron beam of the SEM. The electrically conductive tip used thus far is made of boron-through-doped diamond. A cube corner tip geometry was chosen for the experiments presented here. This geometry allows for easy observation of the indenter surroundings during the experiment.

Electrostatic actuation of the nanoindenter probe is realized by applying a known voltage between the movable center plate and the tip-side fixed outer plate of the force actuator sensor core (Fig. 1, IIb). In general, the electrostatic force $F_{\mathrm{e}}$ generated by an actuatable parallelplate capacitor is described by

$$
F_{\mathrm{e}}=\frac{\kappa_{\mathrm{o}}}{\left(1-\delta / d_{\mathrm{o}}\right)^{2}} V^{2}
$$

where $\kappa_{\mathrm{o}}$ is the zero-volt (resting) electrostatic force constant, $d_{\mathrm{o}}$ is the zero-volt (resting) electrode gap, $\delta$ is the displacement of the center electrode away from $d_{\mathrm{o}}$, and $V$ is the voltage applied across the center plate and the tip-side outer plate. During the indentation test, the electrode gap dimension changes. This has an effect on the scaling between $F_{\mathrm{e}}$ and $V^{2}$ and thus on the true applied electrostatic force. Equation (1) takes into account this electrode gap change during indentation.

Displacement sensing is done using a differential capacitance half-bridge method involving a high-frequency 
$(\sim 150 \mathrm{kHz})$ signal on one fixed outer plate of the displacement sensor core and a second signal of the same frequency but phase shifted by $180^{\circ}$ on the other fixed outer plate of the same core. These signals are high enough in frequency relative to the mechanical bandwidth of the transducer to not actuate it. In the absence of stray capacitance, the transfer function for the displacement signal is:

$$
\frac{V_{\mathrm{out}}}{V_{\mathrm{ac}}}=\frac{C_{2}-C_{1}}{C_{1}+C_{2}}=\frac{\delta}{\bar{d}},
$$

where $V_{\text {ac }}$ is the equal amplitude of the two applied highfrequency signals, $V_{\text {out }}$ is the voltage output from the center plate of this core, and $\bar{d}$ is the mean electrode gap of this core. The voltage output from the center plate will be of the same frequency as the applied high-frequency signals, and its phase $\left(0\right.$ or $\left.180^{\circ}\right)$ will be dictated by whichever of the two capacitances, $C_{1}$ or $C_{2}$, is larger.

The SEM nanoindentation instrument uses a digital signal processor (DSP) based feedback control system in communication with a computer through a universal serial bus (USB) link. This approach was preferred over traditional data acquisition hardware to eliminate plug-in data acquisition cards internal to the computer and to increase portability. The DSP architecture is flexible and ideally suited to handle the real-time data acquisition and control processes of the instrument. In its current configuration, the control system operates in two modes: (i) open-loop load ramp where a predetermined "total applied load" (actually $V^{2}$ ) versus time function is established and (ii) displacement control with a displacement set point versus time ramp. The load applied to the sample is the difference between the total applied load (the electrostatic force) and the force required to displace the springs internal to the transducer $(k \delta$ where $k$ is the effective spring constant of the internal springs). The second mode is a closed-loop mode that uses a proportional-integral-derivative (PID) loop to maintain the predetermined displacement versus time ramp. When operating in the displacement-control mode, the controller also provides for active damping of system vibrations, which is advantageous for the inherently low-damping vacuum environment.

Figure 2 shows the overall computer-aided design (CAD) of the instrument. The main frame is made of titanium, and its lower part (Fig. 2, no. 1) provides support for the $x, y$ (Fig. 2, no. 7) and $z$ (Fig. 2, no. 6) piezoactuators (SmarAct, Oldenburg, Germany) that are used for lateral sample positioning $(x, y)$ and coarse tipsample approach $(z)$. The slip-stick piezoactuators allow for positioning within an accuracy of $50 \mathrm{~nm}$ and have a travel range of several $\mathrm{mm}$. The coarse approach mode is able to bring the tip to within $\sim 100 \mathrm{~nm}$ of the sample surface. Prior to executing the indentation cycle, the

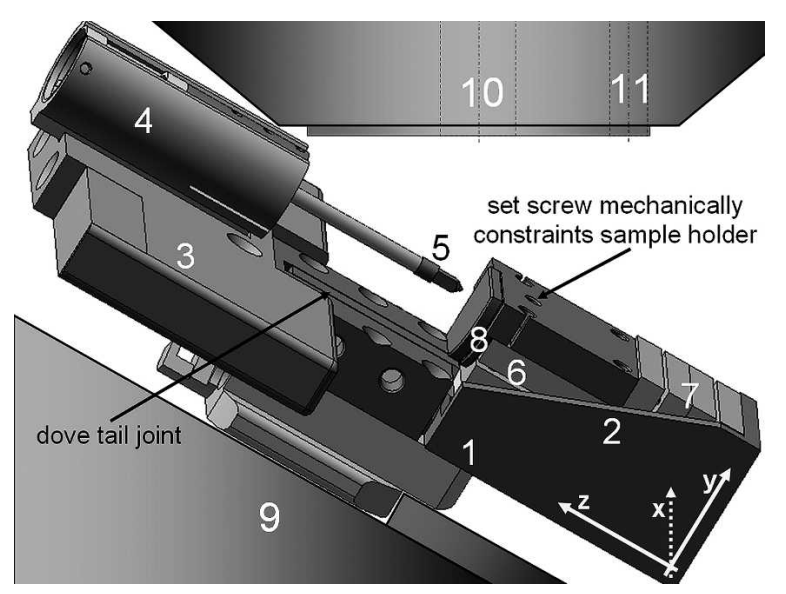

FIG. 2. CAD image of the Hysitron force-displacement transducer integrated inside the Hitachi S-4800 high-resolution SEM. The X-Y-Z coordinate system is related to the PicoIndenter. Note the transducer/ sample is tilted off-axis to allow SEM imaging of the sample surface during the indentation cycle. The numbers in the image correspond to the following components: 1, lower part of the main frame; 2, supporting rib; 3, upper part of the main frame; 4 , transducer in its support; 5 , conductive indenter probe; $6, \mathrm{z}$ slip-stick actuator; $7, \mathrm{x}, \mathrm{y}$ slip-stick actuators; 8 , removable sample holder; 9, SEM stage; 10, SEM electron gun; and 11, SEM high-magnification detector.

force-displacement transducer serves as a fine-approach actuator and brings the tip into initial contact with the sample. The maximum total displacement for the fine approach and indentation cycle is currently $5 \mu \mathrm{m}$. To achieve high structural rigidity, the lower part of the main frame (Fig. 2, no. 1) is supported by a rib on one side (Fig. 2, no. 2). The bending moment, which is a result of the weight of the instrument as well as the force applied during the indentation test, is concentrated at this point.

The sample holder (Fig. 2, no. 8) is supported by a plate to minimize compliance during the indentation test. The shaft of the sample holder is keyed, and a mechanical stop prevents rotation during the indentation cycle. A set screw mechanically constrains the sample holder and locks it in place, preventing additional movement. A conductive silver paste is used for affixing samples to the sample holder to avoid sample charging, which can dramatically reduce SEM image resolution. To facilitate quick and easy tip and sample replacement, the main frame was made in two pieces (lower and upper parts) joined with an adjustable dovetail joint and secured by two set screws. The adjustable dovetail joint also allows for manual adjustment of the distance between the end of the tip and the sample. This accommodates samples of varying heights between 0 to $10 \mathrm{~mm}$.

The upper part of the main frame (Fig. 2, no. 3) has two design functions: (i) provide support for the forcedisplacement transducer and associated electronic circuit boards and (ii) allow for proper relative positioning of the tip and the sample. The support for the forcedisplacement transducer is designed to fix the position of 
the transducer, protect it against the electromagnetic field, and prevent mechanical interference with the other internal components of the SEM.

\section{B. Instrument calibration}

The force-displacement transducer is precalibrated via dead weights and laser interferometry to determine the effective spring constant $k$, the displacement sensitivity, the load sensitivity, and an initial estimate of the electrostatic actuation parameters. Before indentation experiments and at regular intervals, the zero-volt electrostatic force constant $\kappa_{\mathrm{o}}$ and the zero-volt electrode gap $d_{\mathrm{o}}$ are refined by performing a large displacement $(>3 \mu \mathrm{m})$ "airindent" with the tip well removed from the sample $\left(F_{\mathrm{e}}=\right.$ $k \delta$ must hold true at all data points in this situation). Refining the calibration of the electrostatic actuation parameters is crucial because the initial position of the center plate of the force actuator is affected by the tilt angle of the entire nanoindenter in the SEM, which affects both $\kappa_{\mathrm{o}}$ and $d_{\mathrm{o}}$ values.

Two other important factors known to influence nanoindentation results are the machine compliance of the instrument and the tip area function calibration; however, neither has been quantified yet. The primary interest at this stage of the development is to conduct phenomenological studies rather than to determine hardness and elastic modulus. In regard to identifying the potentially significant sources of machine compliance, finite element analysis of the main frame is currently underway, and the overall compliance of the slip-stick actuators used for positioning is currently being evaluated. As for the tip-area function calibration, an attractive option would be to take advantage of the in situ imaging capability of the SEM to provide a direct measurement of the projected contact area at each point of the indentation test. This would allow one to account for pileup or sinkin as well as fracture. The traditional Oliver and Pharr ${ }^{9}$ analysis is not able to directly account for these phenomena.

\section{RESULTS AND DISCUSSION}

\section{A. In situ instrument performance}

The performance of the force-displacement transducer as well as SEM-related factors influence the overall performance of the system. Important factors include the noise level of the transducer, SEM resolution, sample/tip contamination, charging during SEM imaging, and system vibrations. The force-displacement transducer has a maximum load of $\sim 1500 \mu \mathrm{N}$, a load noise floor (RMS) of $\sim 0.1 \mu \mathrm{N}$, a maximum displacement of $5 \mu \mathrm{m}$, and a displacement noise floor (RMS) of $\sim 0.4 \mathrm{~nm}$. The microscope used for integration is a high-resolution Hitachi S-4800 scanning electron microscope. SEM resolution is strongly dependent on the sample material properties (e.g., conductive, semiconductive, or insulative) and the atomic mass of the studied materials. A conductive, boronthrough-doped diamond tip having a cube corner geometry was used for the nanoindentation tests. Both the tip and the sample were grounded to suppress charging. Nevertheless, contact between the tip and the sample often caused a change in image contrast and image resolution in the material around the indent. This is due to local charging and subsequent charge dissipation/ redistribution resulting from the tip-sample contact. Contamination caused by prolonged exposure of the tip and the sample to the electron beam also must be minimized. Carbon deposition on the tip and the sample can result in reduced image resolution.

\section{B. In situ nanoindentation}

Initial data from two different materials are shown below to illustrate the system performance. Interesting observations were obtained from an aluminum $\langle 100\rangle$ single crystal and a thin gold film $(\sim 225 \mathrm{~nm})$ sputter coated onto a fused quartz substrate. Figure 3 shows two load-displacement curves for aluminum, one taken under displacement control [Fig. 3(a)] and the other under open-loop load control [Fig. 3(b)]. The load-drop and pop-in events mostly result from dislocation bursts ${ }^{2}$ occurring in the sample volume beneath the probe during displacement-controlled or open-loop load-controlled nanoindentation, respectively.

As has been shown previously, the displacementcontrol mode tends to be more sensitive to discrete events ${ }^{10}$ as indicated by the clear load drops in Fig. 3(a). The first dramatic load-drop event occurred around $20 \mu \mathrm{N}$ in the case of the displacement-controlled test. This load-drop event may be correlated with breaking through an outer layer atop the substrate, ${ }^{11}$ although dislocation activity in the aluminum is likely to accompany this event. Note how the load drops to negative values after the initial breakthrough event. This is most likely caused by the exposure of bare aluminum, which has a much higher surface energy than the oxide outer surface and results in a significant adhesive interaction. ${ }^{2} \mathrm{~A}$ similar transition from oxide-indenter to metal-indenter interaction was seen during displacement control mode indents on indium. ${ }^{10}$ The adhesive interaction may be further enhanced by the relatively clean vacuum environment and minimal water adsorption layers. The large adhesive force observed upon unloading also is indicative of a bare-metal-indenter interaction.

For comparison, the same type of experiment was performed in the open-loop load-control mode with which pop-in events ${ }^{12}$ were observed [Fig. 3(b)]. This behavior was confirmed by the SEM video recording taken during this experiment, which showed the indenter tip making a sudden jump into the material ${ }^{13}$ [see Fig. 3(b) insets]. No 


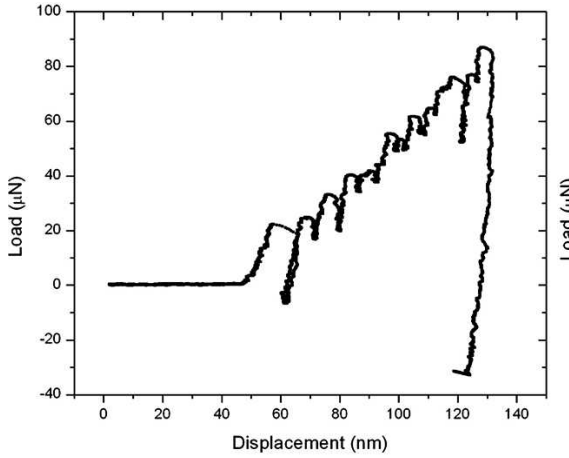

(a)

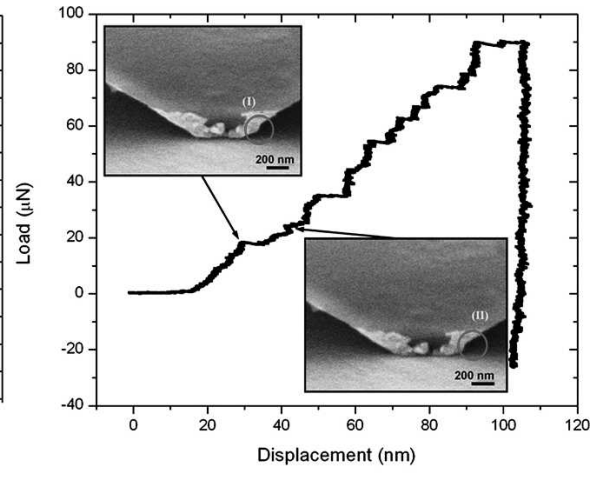

(b)

FIG. 3. In situ SEM nanoindentation of an aluminum $\langle 100\rangle$ single crystal. Load-displacement curves acquired in two different modes: (a) displacement control mode and (b) open-loop load control mode. The insets in (b) are the SEM images that correspond to the indicated points on the load-displacement plot and demonstrate a rapid change in the indentation depth caused by breaking through the outer layer. The circles help to distinguish the difference between the two images.

pileup or sink-in was observed within the resolution limits of the SEM. The outer layer material was often found to delaminate from the aluminum and adhere to the indenter tip [see Fig. 3(b) insets]. This illustrates the need for in situ observation to detect, observe, and correct for these events.

Thin gold films are often used as substrates for the formation of self-assembled monolayers and other molecular thin films. ${ }^{14,15}$ Gold is inert, yet can be easily modified through the chemisorption of molecules containing a thiol or disulfide functional group. The roughness of the gold film as well as adhesion to the underlying substrate can be critical to achieving the ultimate goal of a well-characterized functional surface used for a variety of applications (e.g., molecular/organic electronics, biofunctional surfaces). Gold films have also been used as electrodes in electrochemical studies and applications. ${ }^{16}$ The gold film can be evaporated onto the quartz surface of a quartz crystal microbalance. A thin metallic layer (e.g., titanium or chromium) is often used to promote the adhesion of metallic layers to the underlying substrate. Without the adhesion layers, the films are often only weakly adsorbed and can easily delaminate, limiting the practical use of the films. However, this adhesion layer can lead to deleterious effects such as influencing the surface roughness or actually dissolve into solution under certain potentials as was shown by Hoogvliet and van Bennekom. ${ }^{16}$

Figure 4 shows the results from in situ SEM nanoindentation on a sample consisting of a thin gold film $(\sim 225 \mathrm{~nm})$ sputter coated onto a fused quartz substrate. No interfacial layer was used to promote adhesion of the gold film to the substrate. The absence of the adhesion layer resulted in a weak interfacial bond as was evident from the data. The nanoindentation test was performed under displacement control (see the movie in the supplemental information). There was significant adhesion be- tween the tip and the indented material as indicated in the load-displacement curve [Fig. 4(a) inset], similar to the aluminum data shown previously. The tip-sample adhesion was strong enough to cause delamination of the film from the substrate. The SEM images in Figs. 4(d) and 4(e) suggest that the film actually lifts off of the substrate and then releases from the tip. The real-time movie of this indentation provides a better depiction of the delamination event than the still images alone (see the movie in the supplemental information). This delamination event explains the discontinuous shape of the unload portion of the load-displacement curve shown in the inset of Fig. 4(a). Further evidence for this mechanism would require the time-consuming process of obtaining a cross section of the residual indent to visualize the interfacial crack. The in situ SEM observation provided clear evidence of film delamination and allowed for accurate interpretation of the load-displacement curve. Without the SEM observation, one may erroneously interpret the load-displacement curve as simple adhesion between the tip and film. This is a clear example of the utility provided by the real-time imaging in concert with the load-displacement data.

\section{CONCLUSION}

The SEM nanoindentation system described previously illustrates the power of combining quantitative load-displacement measurement with real-time SEM imaging. On the aluminum $\langle 100\rangle$ sample, the oxide breakthrough event was observed in real-time and easily time correlated to the pop-in event observed in loaddisplacement curves. On the gold film sample, the negative load values and the discontinuity observed in the load-displacement data could be accurately attributed to tip/sample adhesion and film delamination. The real-time imaging provided clear evidence of this mechanism. 


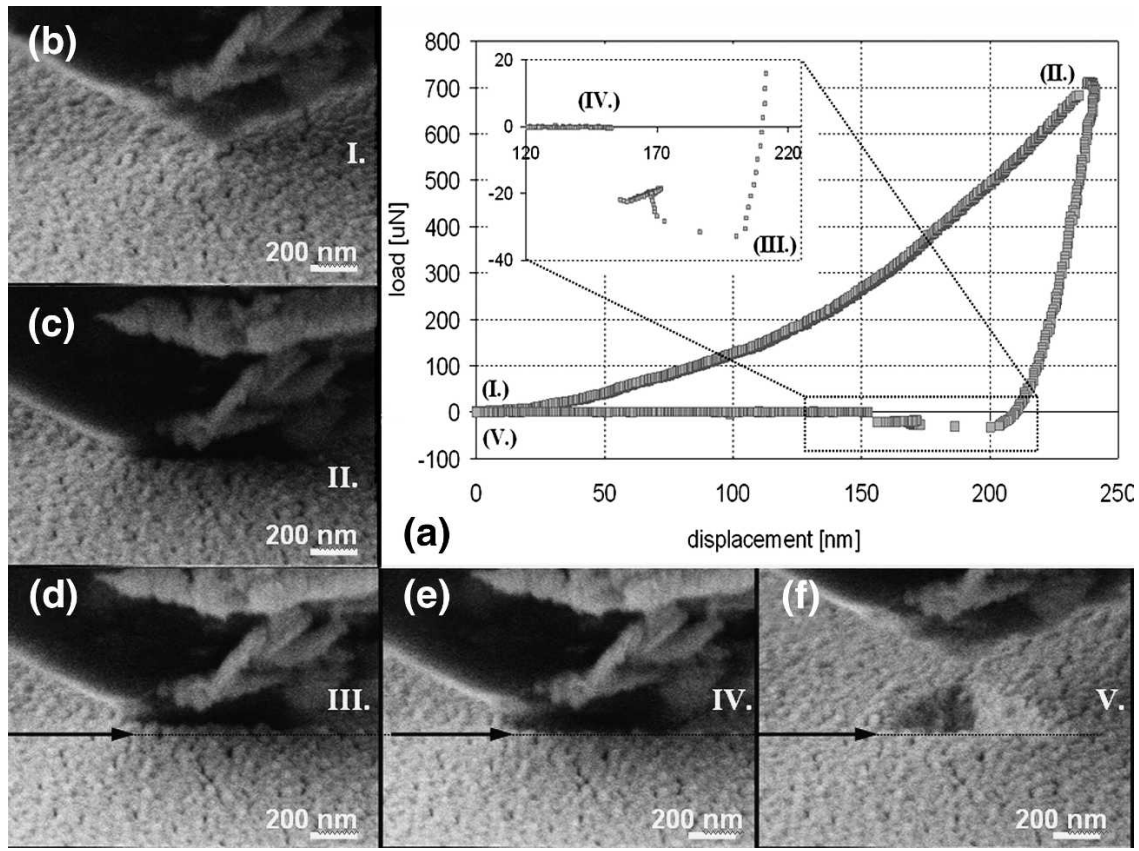

FIG. 4. In situ SEM nanoindentation of a thin gold film $(\sim 225 \mathrm{~nm})$ deposited on fused quartz. The complete load-displacement curve is shown in (a). Images (b-f) were taken from the SEM movie recorded during the indentation test and correspond to the numbered points shown in the curve (a). (b) I corresponds to the initial point of contact; (c) II maximum load; (d) III delamination of the coating from the substrate; (e) IV immediately after film delamination; and (f) V high-resolution image of the residual indent. The reference line and arrows are used to help distinguish the lift-off of the gold film.

Educated speculation about the cause of certain features in the indentation curve may now be supported by simultaneous imaging of the nanoindentation test. The use of high spatial resolution, real-time imaging provides accurate nanopositioning capabilities. In situ testing provides valuable insight into phenomena such as pileup or sink-in, delamination, and other material deformation behavior that may occur during nanoindentation experiments. The SEM imaging also allows for the precise positioning of the indenter probe and selection of a defect free location for the indentation test. This instrument is an ideal tool for the characterization of particularly challenging nanostructured materials such as nanowires, nanopillars, and nanospheres, where instrumentation requirements (i.e., force/displacement resolution, accuracy of the positioning system, and high magnification capabilities) are extremely important.

\section{ACKNOWLEDGMENTS}

The authors thank T.J. Wyrobek for supporting the project. We are grateful to S. Hoesli and E. Pieper for help and discussions regarding design and vacuum compatibility of mechanical parts and for machining some of them, and to K. Wasmer for fruitful discussions. The force-displacement transducer and associated controller/ software were developed under a United States Department of Energy SBIR grant (No. DE-FG02-04ER83979) awarded to Hysitron, Inc. We thank Swiss State Secretariat for Education and Research, EU FP6 project MASMICRO (No. 03.0225, NMP2-CT-2004-500095) for additional financial support.

\section{REFERENCES}

1. A.C. Fisher: Cripps: Nanoindentation (Springer-Verlag, New York, 2002).

2. A.M. Minor, S.A.S. Asif, Z. Shan, E.A. Stach, E. Cyrankowski, T.J. Wyrobek, and O.L. Warren: A new view of the onset of plasticity during the nanoindentation of aluminum. Nature Mat. $\mathbf{5}$, 697 (2006).

3. N. Gane and F.P. Bowden: Microdeformation of solids. J.Appl. Phvs. 39, 1432 (1968).

4. H. Bangert and A. Wagendristel: Ultralow-load hardness tester for use in a scanning electron microscope. Rev. Sci. Instr. 56(8), 1568 (1985).

5. H. Bangert and A. Wagendristel: Ultralow load hardness testing of coatings in a scanning electron microscope. J. Vac. Sci. Technol. 4(6), 2956 (1986).

6. R. Rabe, J-M. Breguet, P. Schwaller, S. Stauss, F-J. Haug, J. Patscheider, and J. Michler: Observation of fracture and plastic deformation during indentation and scratching inside the scanning electron microscope. Thin Solid Films 206, 469 (2004).

7. J. Michler, R. Rabe, J-L. Bucaille, B. Moser, P. Schwaller, and J-M. Breguet: Investigation of wear mechanisms through in situ observation during microscratching inside the scanning electron microscope. Wear 259, 18 (2005).

8. O.L. Warren, S.A.S. Asif, E. Cyrankowski, and K. Kounev: U.S. Patent Application No. 20070180924 (filed Feb 7, 2007). 
9. W.C. Oliver and G.M. Pharr: An improved technique for determining hardness and elastic modulus using load and displacement sensing indentation experiments. J. Mater. Res. 7,1564 (1992).

10. O.L. Warren, S.A. Downs, and T.J. Wyrobek: Challenges and interesting observations associated with feedback-controlled nanoindentation. Z. Metallkd. 95, 287 (2004).

11. W.W. Gerberich, J.C. Nelson, E.T. Lilleodden, P. Anderson, and J.T. Wyrobek: Indentation induced dislocation nucleation: The initial yield point. Acta Mater. 44, 3585 (1996).

12. Y. Shibutani, T. Tsuru, and A. Koyama: Nanoplastic deformation of nanoindentation: Crystallographic dependence of displacement bursts. Acta Mater. 55, 1813 (2007).
13. B. Moser, J. Kuebler, H. Meinhard, W. Muster, and J. Michler: Observation of instabilities during plastic deformation by in situ SEM indentation experiments. Adv. Eng. Mat. 7(5), 388 (2005).

14. R.G. Nuzzo and D.L. Allara: Adsorption of bifunctional organic disulfides on gold surfaces. J. Am. Chem. Soc. 105, 4481 (1983).

15. A. Ulman: An Introduction to Ultrathin Organic Films from Langmuir-Blodgett to Self-Assembly (Academic Press, San Diego, CA, 1991)

16. J.C. Hoogvliet and W.P. van Bennekom: Gold thin-film electrodes: An EQCM study of the influence of chromium and titanium adhesion layers on the response. Electrochim. Acta 47(4). 599 (2001) 